\title{
Global Analysis of Fragmentation Functions for Protons and Charged Hadrons
}

\author{
Daniel de Florian* and Rodolfo Sassoti \\ Departamento de Fisica, Universidad de Buenos Aires, \\ Ciudad Universitaria, Pab. 1 (1428) Buenos Aires, Argentina \\ Marco Stratmann \\ Radiation Laboratory, RIKEN, 2-1 Hirosawa, Wako, Saitama 351-0198, Japan
}

\begin{abstract}
We present new sets of fragmentation functions for protons and inclusive charged hadrons obtained in combined NLO QCD analyses of single-inclusive hadron production in electron-positron annihilation, proton-proton collisions, and deep-inelastic lepton-proton scattering. These analyses complement previous results for pion and kaon fragmentation functions with charge and flavor discrimination. The Lagrange multiplier technique is used to assess the uncertainties in the extraction of the new sets of fragmentation functions.
\end{abstract}

PACS numbers: 13.87.Fh, 13.85.Ni, 12.38.Bx

\section{INTRODUCTION}

Single-inclusive hadron production is the most appropriate and powerful benchmark for challenging altogether our understanding of the partonic structure of nucleons, the dynamics of hard Quantum Chromodynamics (QCD) interactions, the validity of QCD factorization, and the way in which quarks and gluons produce the detected final-state hadrons. The last few years have witnessed a remarkable improvement in both precision and variety for this kind of measurements, which are expected to continue yielding crucial pieces of information in the future.

In a recent article [1], we have demonstrated the feasibility of performing a next-to-leading order (NLO) combined QCD analysis of single-inclusive pion and kaon production data, coming from electron-positron annihilation, semi-inclusive deep-inelastic lepton-nucleon scattering (SIDIS), and proton-proton collisions, collected over a wide kinematic range. The analysis not only allowed a very good global description of all these processes, but provided also accurate parametrizations for the parton-to-pion and parton-to-kaon fragmentation functions, which encode the details of the non-perturbative hadronization process relevant for the perturbative QCD (pQCD) framework based on the factorization theorem.

In the following, we extend the analysis of Ref. [1] first to the case of single-inclusive proton and anti-proton production, again including not only the electron-positron annihilation data 2, 3, 4, 5, 5, 6] used in all previous fits [7, 8, 9], but, for the first time, also the very precise data recently obtained in proton-proton collisions at RHIC [10], where final state protons and anti-protons are discriminated.

Next, after having obtained reliable fragmentation

\footnotetext{
*Electronic address: deflo@df.uba.ar

${ }^{\dagger}$ Electronic address: sassot@df.uba.ar

‡Electronic address: marco@ribf.riken.jp
}

functions for the three lightest and most copiously produced charged hadron species, pions and kaons from Ref. 1] and (anti-)protons, we analyze inclusive, i.e., unidentified, charged hadron yields obtained in electronpositron annihilation [2, 3, 4, [5, 11, 12, 13, 14], proton(anti-)proton collisions [15, 16, 17], and SIDIS 18]. Here we are aiming at an extraction of the contribution from the "residual" charged hadrons other than pions, kaons, and (anti-)protons, to the inclusive charged hadron fragmentation functions.

Only from a global QCD analysis we can obtain individual quark and anti-quark fragmentation functions for unidentified positively or negatively charged hadrons without having to make assumptions on the relation between favored (valence-type) and unfavored (sea-type) contributions. Such assumptions have been shown to be often not adequate for reproducing presently available single-inclusive hadron production yields beyond those obtained in electron-positron annihilation [1]. In addition, data from proton-(anti-)proton collisions are crucial for reducing the uncertainties in the gluon-tohadron fragmentation function [1] since scaling violations of $e^{+} e^{-}$data are not much of a constraint due to the lack of precision data at more than one value of the center-ofmass system (c.m.s.) energy $\sqrt{s}$. Both resulting new sets of NLO fragmentation functions reproduce with remarkable accuracy the data available and hence complement consistently our previous studies for pions and kaons.

Previously available sets of inclusive charged hadron fragmentation functions are all based on fits to $e^{+} e^{-}$data only and either sum up the results obtained for pions, kaons, and (anti-)protons, ignoring possible contributions from heavier charged hadrons [7, 8] or the residual hadron contribution already includes protons and anti-protons [19]. The analysis of Ref. 20] provides only fragmentation functions for unidentified charged hadrons, again based on $e^{+} e^{-}$data only, however, an attempt was made to further constrain the gluon fragmentation function by comparing to one of the many data sets from proton(anti-)proton collisions. 
In the next section, we briefly highlight the main features of the present analysis, specifically discussing our choice of parametrizations for the proton and the residual charged hadron fragmentation functions and emphasizing the charge conjugation and flavor symmetry assumptions we still have to make. In Section III we present our results for both NLO fits, and assess the uncertainties involved with the help of the Lagrange multiplier technique. In Sec. IV we summarize our main results. For completeness, the Appendix contains the results of the global analyses performed at leading order (LO) accuracy, which compares significantly less favorable to data than our NLO fits.

\section{OUTLINE}

In the present analysis we work in the well established framework of pQCD for single-inclusive hadron production processes at NLO accuracy, thoroughly discussed and implemented in Ref. [1]. We make an extensive use of the Mellin transform technique [21] developed for a fast computation of the exact NLO cross sections in each step of a global $\chi^{2}$ minimization procedure. We refer the reader to [1] and references therein for further technical details on the general QCD framework and the assessment of uncertainties in global fits with the help of Lagrange multipliers. The analysis proceeds in two stages, the first dedicated to the extraction of the parton-to(anti-)proton fragmentation functions, followed by the determination of inclusive charged hadron fragmentation functions in the second.

\section{A. Proton fragmentation functions}

In the first step of our global analysis we aim to determine individual fragmentation functions $D_{i}^{H}$ for quarks and anti-quarks of all flavors $i$ as well as for gluons into either protons $(H=p)$ or anti-protons $(H=\bar{p})$. At variance with previous analyses [7, 8, 9], the use of data that discriminate between $p$ and $\bar{p}$ in the final-state, allows us to extract fragmentation functions for either particle. As explained in Ref. [1], in order to have the flexibility required by charge separated distributions and to accommodate the additional data, we adopt a somewhat more versatile functional form for the input distributions at scale $\mu_{0}$ than in [7, 8, [9]

$$
\begin{aligned}
D_{i}^{H}\left(z, \mu_{0}\right) & = \\
& \frac{N_{i} z^{\alpha_{i}}(1-z)^{\beta_{i}}\left[1+\gamma_{i}(1-z)^{\delta_{i}}\right]}{B\left[2+\alpha_{i}, \beta_{i}+1\right]+\gamma_{i} B\left[2+\alpha_{i}, \beta_{i}+\delta_{i}+1\right]},
\end{aligned}
$$

where $B[a, b]$ represents the Euler beta function and $N_{i}$ is normalized such to represent the contribution of $D_{i}^{H}$ to the second moment $\int_{0}^{1} d z z D_{i}^{H}\left(z, \mu_{0}\right)$ entering the energymomentum sum rule. $z$ denotes the fraction of the fragmenting parton's energy taken by the produced hadron
$H$. As in [1], the initial scale $\mu_{0}$ in Eq. (1) for the $Q^{2}$ evolution is taken to be $\mu_{0}=1 \mathrm{GeV}$ for the light $u, d, s$ partons and the quark masses for the heavier ones.

The parameters describing the fragmentation functions $D_{i}^{H}\left(z, \mu_{0}\right)$ in Eq. (11) are determined by a standard $\chi^{2}$ minimization for $K$ data points, where

$$
\chi^{2}=\sum_{j=1}^{K} \frac{\left(T_{j}-E_{j}\right)^{2}}{\delta E_{j}^{2}},
$$

$E_{j}$ is the measured value of a given observable, $\delta E_{j}$ the error associated with this measurement, and $T_{j}$ is the corresponding theoretical estimate for a given set of parameters in (1). Since the full error correlation matrices are not available for most of the data entering the global analysis, we take the statistical and systematical errors in quadrature in $\delta E_{j}$.

In order to reduce the number of parameters in (1) to those that can be effectively constrained by the data, we impose, as usual, certain relations among the individual fragmentation functions. We have checked in each case that relaxing these assumptions indeed does not significantly improve the total $\chi^{2}$ of the fit (2) to warrant any additional parameters. In detail, for the fragmentation of light quark flavors to a proton we assume the same shape for up and down quarks and for up and down antiquarks with the same $z$-independent normalization ratios $N$, i.e.,

$$
D_{u}^{p}=N D_{d}^{p} \quad \text { and } \quad D_{\bar{u}}^{p}=N D_{\bar{d}}^{p} .
$$

The relation between quark and anti-quark fragmentation functions for $u$ and $d$ flavors is determined by the global fit through

$$
2 D_{\bar{q}}^{p}=(1-z)^{\beta} D_{q+\bar{q}}^{p},
$$

with $\beta$ constrained to be positive. For strange quarks it is assumed that

$$
D_{s}^{p}=D_{\bar{s}}^{p}=N^{\prime} D_{\bar{u}}^{p},
$$

with the $\mathrm{SU}(3)$-breaking parameter $N^{\prime}$ independent of $z$. For charm, bottom, and gluon-to-proton fragmentation we find no improvement in the total $\chi^{2}$ for $\gamma_{i} \neq 0$ in (1), hence we set $\gamma_{i}=0$.

To obtain the corresponding fragmentation functions $D_{i}^{\bar{p}}$ for anti-protons we assume charge conjugation symmetry, i.e.,

$$
D_{q}^{p}=D_{\bar{q}}^{\bar{p}},
$$

leaving in total 17 free parameters to be determined by the global fit.

\section{B. Charged hadron fragmentation functions}

The fragmentation functions $D_{i}^{H}$ for unidentified positively charged hadrons $\left(H=h^{+}\right)$are defined by

$$
D_{i}^{h^{+}}=D_{i}^{\pi^{+}}+D_{i}^{K^{+}}+D_{i}^{p}+D_{i}^{r e s^{+}},
$$


where $D_{i}^{\pi^{+}}$and $D_{i}^{K^{+}}$were already determined in Ref. [1]. $D_{i}^{r e s^{+}}$denotes the residual contribution of positively charged hadrons other than pions, kaons, and protons to the inclusive sum $D_{i}^{h^{+}}$. A definition analogous to Eq. (7) is used for the fragmentation $D_{i}^{h^{-}}$into negatively charged hadrons.

Since pions are much more copiously produced than heavier kaons and protons are even less abundant, it is natural to expect that Eq. (77) is strongly dominated by $D_{i}^{\pi^{+}}+D_{i}^{K^{+}}+D_{i}^{p}$ leaving $D_{i}^{r e s^{+}}$to be small. Nevertheless it is an important consistency check to extract a small but non-vanishing $D_{i}^{\text {res }}{ }^{+}$(and $D_{i}^{\text {res }}{ }^{-}$) from data to actually confirm this hierarchy. If the global fit would require, for instance, a large or even a negative residual contribution, the usefulness of the previously extracted fragmentation functions for light hadrons would be in jeopardy.

For $D_{i}^{r e s^{+}}$it turns out that the data are most economically described by assuming full SU(3) flavor symmetry for both quarks and anti-quarks

$$
D_{u}^{r e s^{+}}=D_{d}^{r e s^{+}}=D_{s}^{r e s^{+}}
$$

and

$$
D_{\bar{u}}^{r e s^{+}}=D_{\bar{d}}^{r e s^{+}}=D_{\bar{s}}^{r e s^{+}},
$$

respectively, in the ansatz (1). Again,

$$
2 D_{\bar{q}}^{r e s^{+}}=(1-z)^{\beta^{\prime}} D_{q+\bar{q}}^{r e s+}
$$

and for $D_{c, b}^{r e s^{+}}$we set $\gamma_{c, b}=0$ in (11), however, $D_{g}^{\text {res }}{ }^{+}$has a preference for $\gamma_{g} \neq 0$. For $D_{i}^{\text {res }}{ }^{+}$we assume charge conjugation symmetry, i.e.,

$$
D_{q}^{\text {rest }}=D_{\bar{q}}^{\text {res- }},
$$

leaving in total 18 free parameters to be fitted here.

We note that the $Q^{2}$-dependence for all fragmentation functions is computed with the appropriate NLO evolution equations as explained in detail in Ref. [1]. Uncertainties in the extraction of the parameters in Eq. (1) will be assessed with the help of the Lagrange multiplier technique as described again in [1] and references therein.

\section{RESULTS OF THE GLOBAL ANALYSES}

In this section we discuss in detail the results of our global analyses of fragmentation functions [31] for protons and residual charged hadrons as outlined above. We present the parameters of the optimum fits describing the NLO $D_{i}^{p, r e s^{+}}$at the input scale $\mu_{0}$ and compare to all data sets used in the analyses, including individual $\chi^{2}$ values. Detailed comparisons are made with previous fits based exclusively on the available electron-positron annihilation data in Refs. [8], [9], and [19], in the following labeled as AKK, HKNS, and KRE, respectively.

\section{A. NLO analysis of proton fragmentation functions}

Three different processes allow the extraction of the (anti-)proton fragmentation functions in our global analysis. First of all, we have the "standard" electronpositron annihilation data [2, 3, 4, 5] customarily included also in most of the previous analyses $[7,[8,9]$. A characteristic feature of $e^{+} e^{-}$annihilation data in general is that they only provide information on a certain hadron species summed over the charges, i.e., in this case on the sum of protons and anti-protons. Besides the fully inclusive measurements, SLD [3] and DELPHI [5] also give results for "flavor enriched" cross sections, distinguishing between the sum of light $u, d, s$ quarks, charm, and bottom events. The quark flavor is determined from Monte-Carlo simulations and refers to the primary $q \bar{q}$ pair created by the intermediate $Z$-boson or photon. Such results have to be taken with a grain of salt as they cannot be unambiguously interpreted and calculated in pQCD. Nevertheless, in our analysis of pion and kaon data [1] we always found good agreement between data and theory.

In Fig. 1 we provide a detailed comparison in terms of "(data-theory)/theory" between all the $e^{+} e^{-}$data sets included in the analysis and our NLO results. The best agreement is found for ALEPH and DELPHI inclusive measurements, although SLD and TPC, as well as flavor tagged data sets, also agree well with the fit within the fairly large experimental uncertainties. The fluctuations in the data are significantly larger than in the case of pions, but roughly comparable to those found for kaons, see Ref. [1]. Other recent fits [8, 9] all reproduce the data shown in Fig. 1 equally well within the experimental uncertainties. Since the current $e^{+} e^{-}$data basically only constrain the total singlet fragmentation function $D_{\Sigma}^{p+\bar{p}}$ at scale $M_{Z}$, all sets differ considerably in their individual flavor content as we shall see below.

We wish to recall that the range of applicability for fragmentation functions is severely limited to mediumto-large values of $z$, see, e.g., Ref. [1]. In order to avoid the potentially problematic low- $z$ region, we exclude from the fit all data with energy fractions lower than $z_{\min }=$ 0.1 . The extrapolation of our fit, however, reproduces the trend of the data reasonably well also below $z_{\text {min }}$ as indicated in Fig. 1.

The second key ingredient in our global analysis are the single-inclusive hadron production data from protonproton collisions at BNL-RHIC taken by STAR [10] at mid-rapidity $|y|<0.5$ and shown in Fig. 2. These data discriminate final-state protons from anti-protons and hence, in principle, allow to separate quark-to-proton and anti-quark-to-proton fragmentation functions in the fit. However, at the presently accessible range of transverse momenta $p_{T}$ and at mid-rapidities the production of single-inclusive hadrons is mainly driven by gluoninduced processes and fragmentation, turning these data into the best constraint on the gluon fragmentation function $D_{g}^{p}$ at large values of $z$ currently available. Figure 


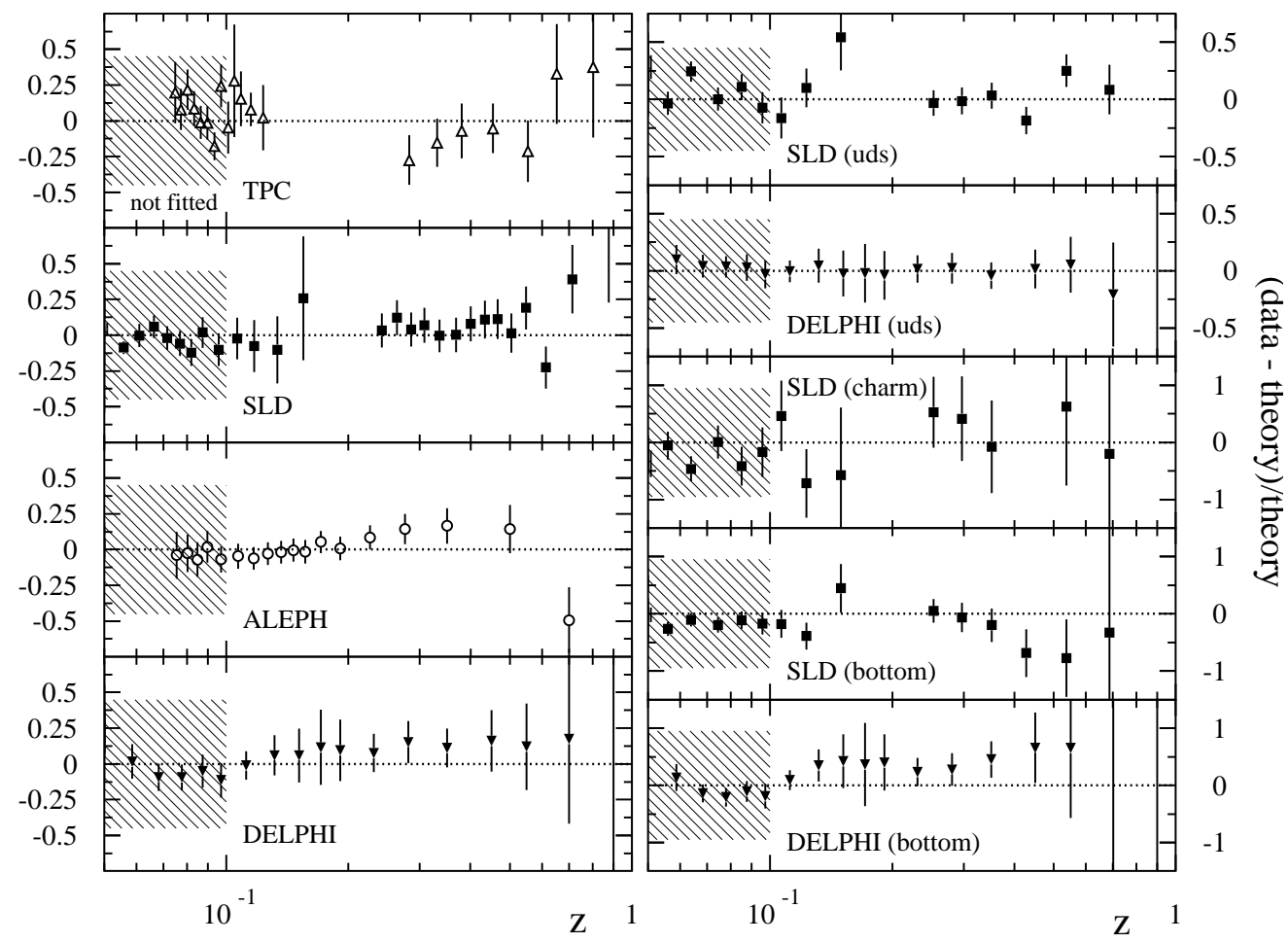

FIG. 1: "(data-theory)/theory" comparison of our NLO results for the electron-positron annihilation cross section into protons and anti-protons with the data sets used in the fit, see also Tab. II

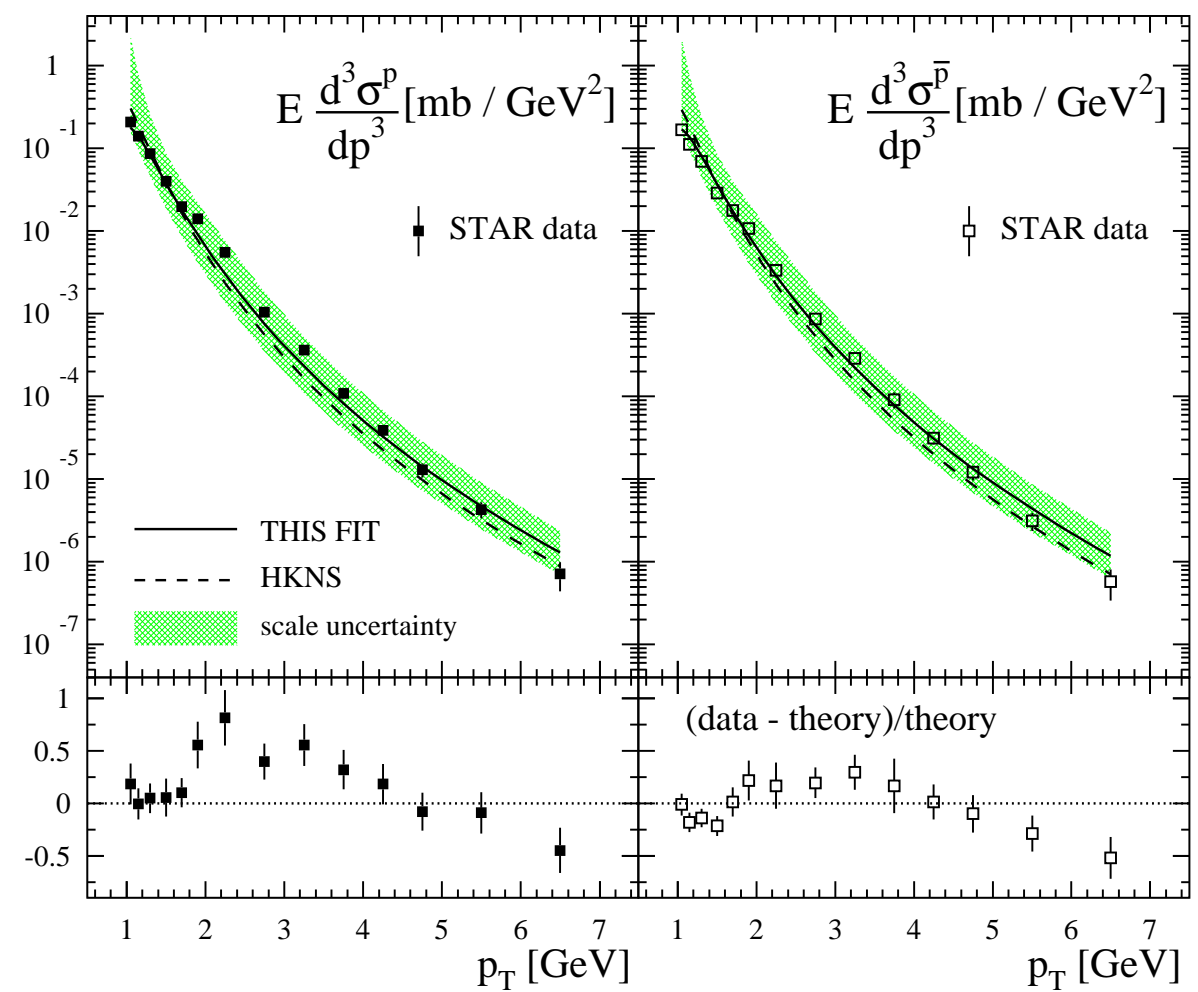

FIG. 2: Upper panels: comparison of our NLO results (solid lines) for single-inclusive proton (anti-proton) production $p p \rightarrow$ $p(\bar{p}) X$ at $\sqrt{s}=200 \mathrm{GeV}$ with STAR data [10] at mid-rapidity $|y|<0.5$ using $\mu_{f}=\mu_{r}=p_{T}$. Also shown are the results obtained with the HKNS [9] parametrization. The shaded bands indicate theoretical uncertainties when all scales are varied in the range $p_{T} / 2 \leq \mu_{f}=\mu_{r} \leq 2 p_{T}$. Lower panels: "(data-theory)/theory" for our NLO results. 


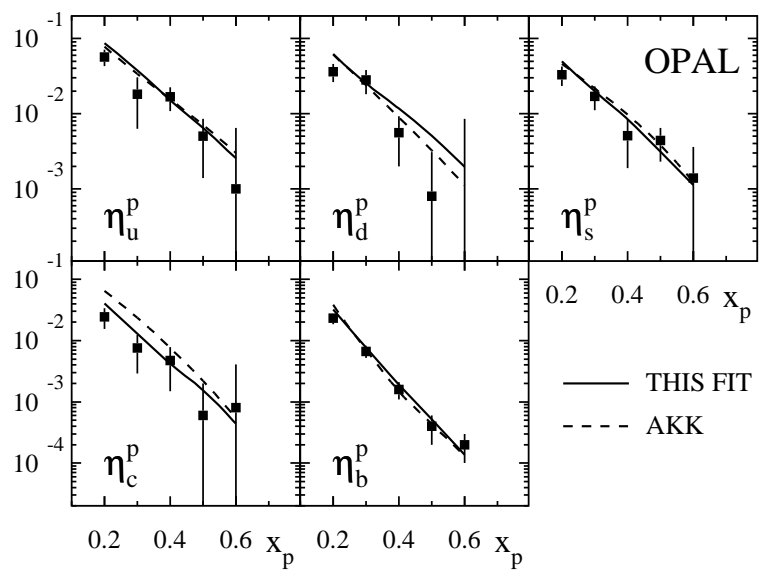

FIG. 3: Comparison of the OPAL "tagging probabilities" [6] for protons plus anti-protons, as a function of the minimum $x_{p}$, with our NLO results (solid lines). Also shown are the results obtained with the AKK [8] parameterization (dashed lines).

2 shows the results of the fit compared to the data. The theoretical uncertainties related to a particular choice of the factorization and renormalization scales, $\mu_{f}$ and $\mu_{r}$, respectively, in the computation of the NLO cross section, indicated by the shaded bands, are non-negligible. This has been taken into account in the $\chi^{2}$ minimization procedure as a conservative additional $5 \%$ relative uncertainty.

We have checked that by excluding data at low $p_{T}$ values, $p_{T} \lesssim 3 \mathrm{GeV}$, the results of the global fit remain essentially unchanged. The fact that the $p_{T}$ spectrum is well reproduced down to $1 \mathrm{GeV}$, the mass of the proton, may turn out to be accidental, but this can only be verified once data at higher $p_{T}$ become available to map out the $p_{T}$ slope more precisely. We note that the BRAHMS data 22] at very forward rapidities $y \simeq 3$ and $p_{T} \lesssim 4 \mathrm{GeV}$ have not been included in our global analysis. These data show a very pronounced difference between the $p$ and $\bar{p}$ yields which cannot be understood. The origin of the excess of protons over anti-protons by a factor of about 10, not observed by STAR at less extreme kinematics, remains an open question 22].

Finally, a third source of information on the parton-toproton fragmentation is provided by the OPAL "tagging probabilities" $\eta_{i}^{p}$ [6], also included in the AKK fit [8] but not in [9], which are sensitive to the flavor of $q+\bar{q}$ fragmentation functions. The $\eta_{i}^{p}\left(x_{p}\right)$ represent "probabilities" for a quark flavor $i$ to produce a "jet" containing the (anti-)proton with a momentum fraction $z$ larger than $x_{p}$. Figure 3 compares the results from our and the AKK fit with the data. As discussed at length in Ref. [1] and also mentioned in the discussion of the flavor tagged SLD and DELPHI data above, any flavor tagged information is highly model dependent and difficult to interpret within pQCD beyond the LO approximation involve non trivial theoretical uncertainties. To take this into account, we assign an up to $10 \%$ extra normalization uncertainty to the $\eta_{i}^{p}$ data in the $\chi^{2}$ minimization. The agreement between OPAL data and theory is reasonably good.

Tables \and II show the values obtained for the parameters in Eq. (11) specifying the optimum fit of proton fragmentation functions $D_{i}^{p}\left(z, \mu_{0}\right)$ at NLO accuracy and summarize the $\chi^{2}$ values for each individual set of data included in the global analysis, respectively. In cases where the normalization uncertainty of the experiment is not included in the error bars of the data, we apply a free normalization factor constrained to vary within range quoted by the experiment. The values of the normalization factors resulting from the fit are also included in Tab. [II]

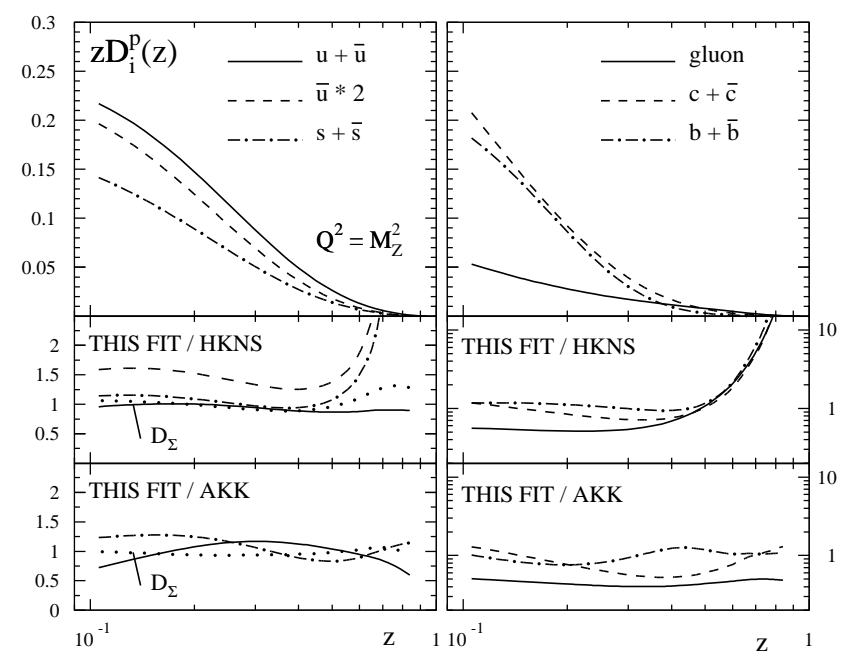

FIG. 4: Upper panels: individual fragmentation functions for protons $z D_{i}^{p}\left(z, Q^{2}\right)$ at $Q^{2}=M_{Z}^{2}$. Middle panels: ratios of our fragmentation functions to the ones of HKNS [9]. The dotted line indicates the ratio for singlet combination of fragmentation functions $z D_{\Sigma}^{p}$. Lower panels: ratios of our fragmentation functions to the ones of AKK [8].

Figure 4 shows the resulting new set of fragmentation functions for protons for different flavors, evolved to the scale of the mass of the Z-boson, and in the middle and lower panels, comparisons to the fits of HKNS [9] and AKK [8], respectively. As expected, the present fit agrees well in the singlet combination $D_{\Sigma}^{p}$ with previous extractions based only on electron-positron annihilation data, but we find significant differences in the gluon fragmentation function, constrained in our fit not only by the scale dependence of the data but also by the STAR data shown in Fig. 2, In addition, there is a sizable difference between our $D_{\bar{u}}^{p}$ and that of HKNS which is mainly a consequence of the $\mathrm{SU}(3)$ flavor symmetry imposed for the unfavored fragmentation functions in the latter analysis [9].

As in Ref. 1], we make use of the Lagrange multiplier technique [23] in order to give a representative picture of the typical uncertainties characteristic of the fragmentation functions obtained from the global fit. In Fig. 5 we show the $\chi^{2}$-profiles as a function of the range of varia- 
TABLE I: Parameters describing the NLO fragmentation functions for protons, $D_{i}^{p}\left(z, \mu_{0}\right)$, in Eq. (1) at the input scale $\mu_{0}=1 \mathrm{GeV}$. Inputs for the charm and bottom fragmentation functions refer to $\mu_{0}=m_{c}=1.43 \mathrm{GeV}$ and $\mu_{0}=m_{b}=$ $4.3 \mathrm{GeV}$, respectively.

\begin{tabular}{cccccc}
\hline \hline flavor $i$ & $N_{i}$ & $\alpha_{i}$ & $\beta_{i}$ & $\gamma_{i}$ & $\delta_{i}$ \\
\hline$u+\bar{u}$ & 0.091 & -0.222 & 1.414 & 15.0 & 3.29 \\
$d+\bar{d}$ & 0.058 & -0.222 & 1.414 & 15.0 & 3.29 \\
$\bar{u}$ & 0.034 & -0.222 & 2.024 & 15.0 & 3.29 \\
$\bar{d}$ & 0.022 & -0.222 & 2.024 & 15.0 & 3.29 \\
$s+\bar{s}$ & 0.043 & -0.222 & 2.024 & 15.0 & 3.29 \\
$c+\bar{c}$ & 0.076 & -0.899 & 5.920 & 0.0 & 0.00 \\
$b+\bar{b}$ & 0.044 & -0.034 & 10.000 & 0.0 & 0.00 \\
$g$ & 0.014 & 6.000 & 1.200 & 0.0 & 0.00 \\
\hline \hline
\end{tabular}

TABLE II: Data used in the NLO global analysis of proton fragmentation functions, the individual $\chi^{2}$ values for each set, the fitted normalizations, and the total $\chi^{2}$ of the fit.

\begin{tabular}{lcccc}
\hline \hline experiment & data & rel. norm. & data points & $\chi^{2}$ \\
& type & in fit & fitted & \\
\hline TPC [2] & incl. & 1.06 & 12 & 7.7 \\
SLD [3] & incl. & 0.983 & 18 & 12.0 \\
& "uds tag" & 0.983 & 9 & 10.5 \\
& "c tag" & 0.983 & 9 & 9.8 \\
& "b tag" & 0.983 & 9 & 8.9 \\
ALEPH [4] & incl. & 0.97 & 13 & 11.5 \\
DELPHI [5] & incl. & 1.0 & 12 & 3.9 \\
& "uds tag" & 1.0 & 12 & 0.6 \\
& "b tag" & 1.0 & 12 & 9.1 \\
OPAL [6] & " $u$ tag" & 1.10 & 5 & 7.6 \\
& " $d$ tag" & 1.10 & 5 & 13.5 \\
& "s tag" & 1.10 & 5 & 5.0 \\
& "c tag" & 1.10 & 5 & 4.9 \\
& "b tag" & 1.10 & 5 & 5.5 \\
\hline STAR [10] & $p$ & 0.95 & 14 & 35.4 \\
& $\bar{p}$ & 0.95 & 14 & 26.0 \\
\hline \hline TOTAL: & & & 159 & 171.9 \\
\hline \hline
\end{tabular}

tion of the truncated second moments of the individual fragmentation functions of flavor $i$,

$$
\eta_{i}^{p}\left(x_{p}, Q^{2}\right) \equiv \int_{x_{p}}^{1} z D_{i}^{p}\left(z, Q^{2}\right) d z
$$

for $x_{p}=0.2$ and $Q=5 \mathrm{GeV}$, around the values obtained for them in the best fit to data, $\eta_{i 0}^{p}$. Roughly speaking, the typical uncertainties in the second moments of the proton fragmentation functions range between $20 \%$ and $25 \%$, allowing for a conservative increase $\Delta \chi^{2}$ of $2 \%$ in the total $\chi^{2}$ of the fit, except for the gluon and bottom fragmentation functions, where the uncertainties are

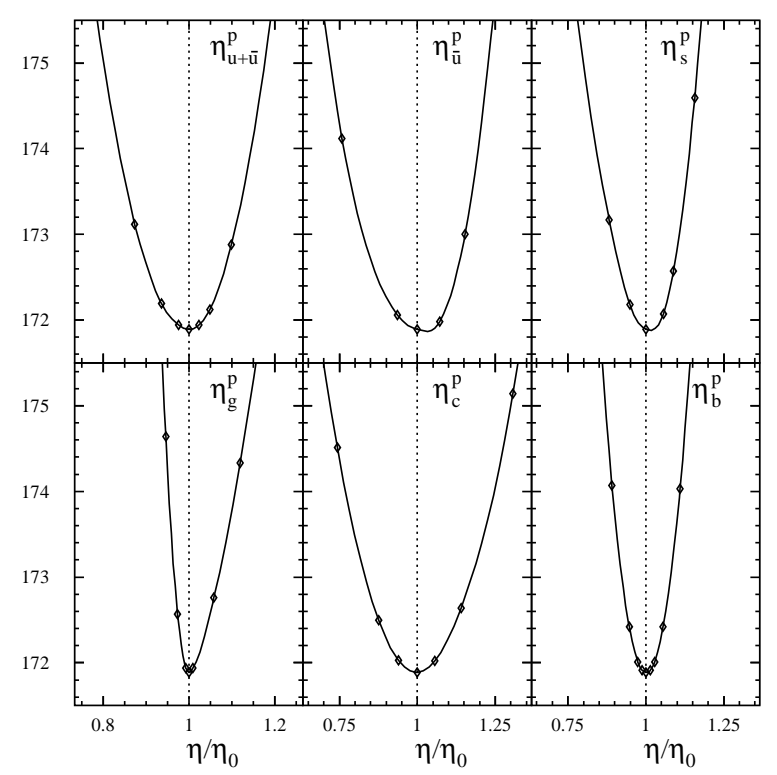

FIG. 5: Profiles of $\chi^{2}$ for the NLO proton fragmentation fit as a function of the truncated second moments $\eta_{i}^{p}\left(x_{p}=\right.$ $0.2, Q^{2}=25 \mathrm{GeV}^{2}$ ) for different flavors. The moments are normalized to the value $\eta_{i 0}^{p}$ they take in the best fit to data.

closer to $10 \%$. The rather stringent constraint for bottom comes from the availability of DELPHI and SLD flavor tagged data, while for charm only SLD data with larger errors are available. Note that neither charm nor bottom fragmentation play any role in the description of the STAR data due to the relatively low scales of $\mathcal{O}\left(p_{T}\right)$ involved. For the gluon fragmentation function $D_{g}^{p}$, the STAR data make the difference. We wish to stress that compared to pions and kaons [1], the parton-to-proton fragmentation functions $D_{i}^{p}$ are much less constrained at the moment. This is also reflected in the stronger assumptions, Eqs. (3)-(5), which have to be imposed on the fit in order to be able to determine all parameters. In particular the lack of (anti-)proton production data from SIDIS prevent a more reliable separation of favored and unfavored fragmentation functions. Also the gluon fragmentation is currently mainly determined from a single set of data (STAR). Here, possible future high precision data from B-factories would open up the possibility for studies of scaling violations in $e^{+} e^{-}$annihilation, which should help to further constrain $D_{g}^{p}$.

\section{B. NLO analysis of charged hadrons fragmentation functions}

As outlined in Sec. IIB, rather than extracting fragmentation functions for inclusive charged hadrons from scratch, we take advantage of the knowledge already acquired on pion, kaon, and proton fragmentation functions. In the following, we isolate and determine the 

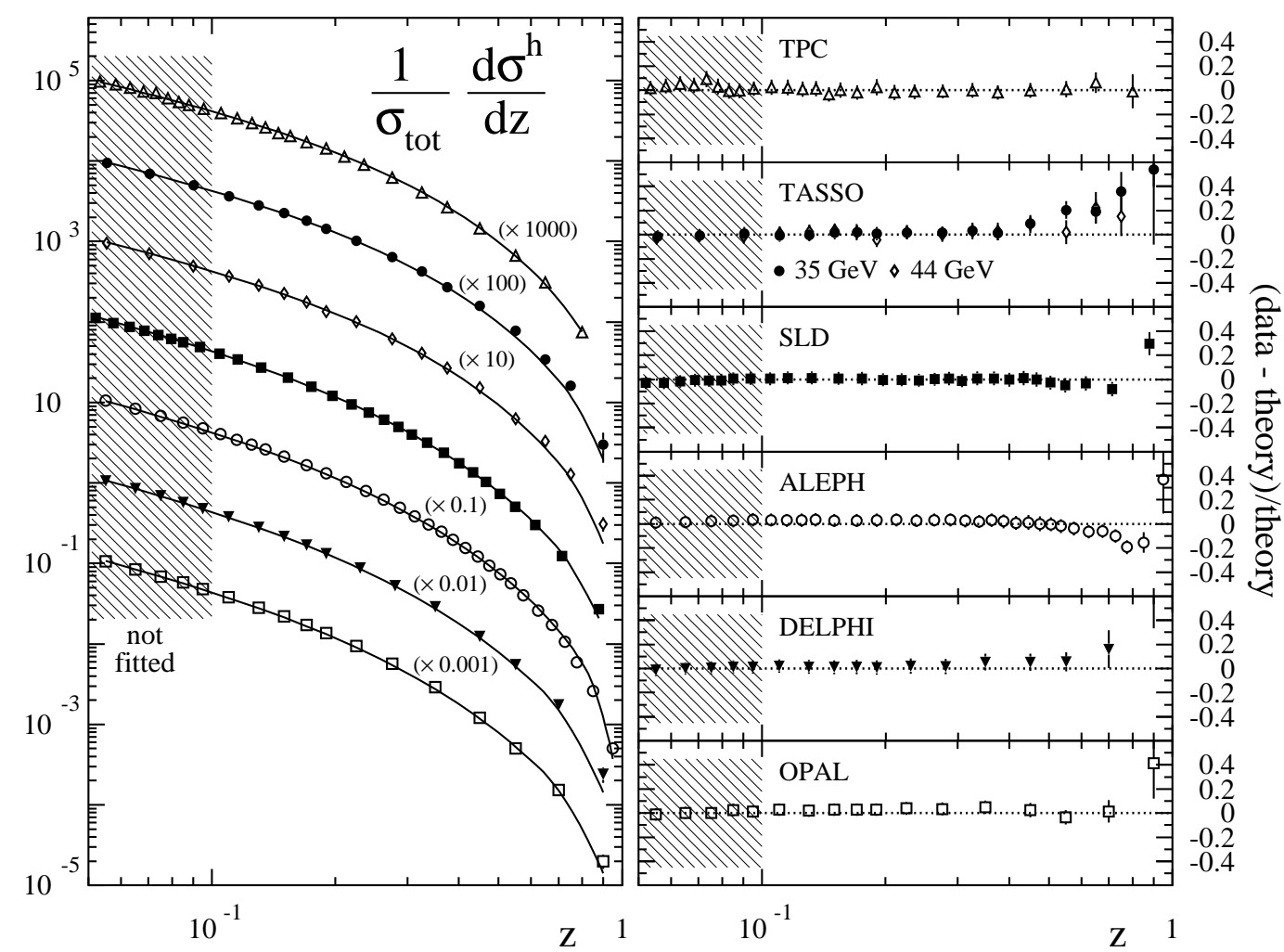

FIG. 6: Left-hand side: comparison of our NLO results for the electron-positron annihilation cross section into inclusive charged hadrons with the data sets used in the fit, see Tab. IV] Right-hand side: (data-theory)/theory for our NLO results for each of the data sets.
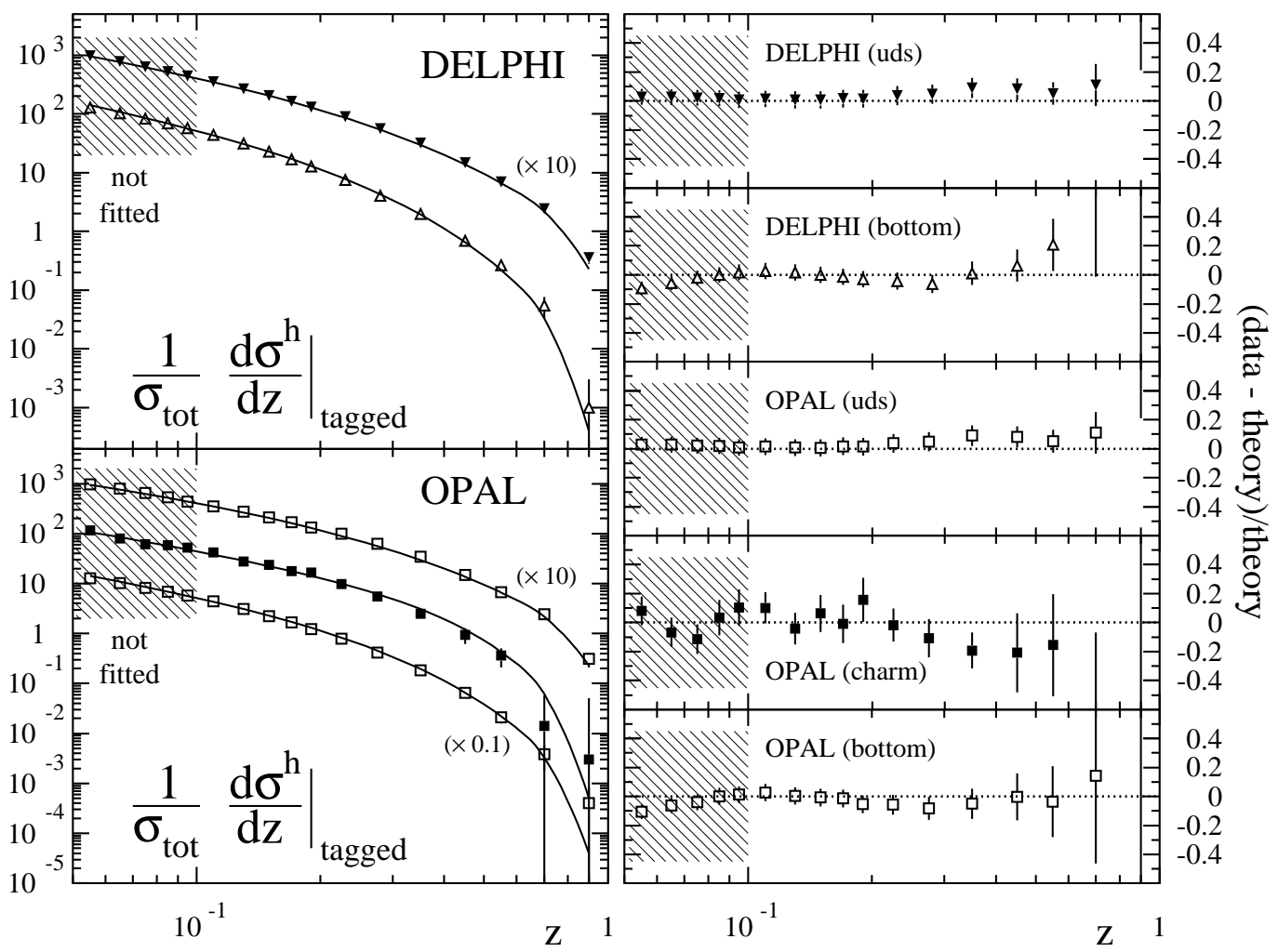

FIG. 7: Same as in Fig. 6 but now for light ("uds") and heavy quark ("c,b") tagged cross sections. 
contributions coming just from residual charged hadrons $D_{i}^{r e s^{ \pm}}$as defined in Eq. (7). In this way, there is no need to make assumptions on the relations between the inclusive charged hadron fragmentation functions - necessary for fitting purposes - which may hold for one of the most abundant hadron species like pions, but not necessarily for the others. Factoring out the contributions from charged pion, kaon, and (anti-)proton fragmentation functions, we are left with a comparatively small residual contribution $D_{i}^{r e s^{ \pm}}$, whose flavor symmetry assumptions, see Sec. IIB have very little impact on the inclusive charged hadron fragmentation functions and the quality and reliability of the fit.

We begin by discussing the remarkable measurements of single-inclusive charged hadron production in $e^{+} e^{-}$annihilation, collected by a variety of experiments $2,2,3,4$, 5, 11, 12]. Figures 6 and 7 show detailed comparisons between the available data and the results of adding the already known pion, kaon, and proton fragmentation functions to the outcome of the fit for the residual hadron contributions $D_{i}^{r e s}{ }^{ \pm}$.

Tables III and IV] show the values obtained for the parameters in Eq. (11) specifying the optimum fit of residual charged hadron fragmentation functions $D_{i}^{r e s}{ }^{+}\left(z, \mu_{0}\right)$ at NLO accuracy and summarize the $\chi^{2}$ values for each individual set of data included in the fit, respectively. As in Tab. III for each set of data, values of the normalization factors resulting from the fit and constrained to vary within range quoted by experiment, are also given in Tab. IV.

All data sets shown in Figs. 6 and 7 are very well reproduced by our new fit, again closely following the trend of the data again also below $z_{\min }=0.1$. This is most apparent in the (data-theory)/theory comparisons. The impressive quality of the fit serves as an important crosscheck of the consistency of entire fitting procedure for all hadron species since the main contributions to the inclusive charged hadron cross sections in Figs. 6 and 7 are already fixed by the pion, kaon, and proton fits. For instance, overshooting the data by just summing up pion, kaons, and protons would have pointed to some serious inconsistency in the global analysis. As expected, we also find that the residual contribution from $D_{i}^{\text {res }}{ }^{ \pm}$is indeed small and - another non-trivial check - positive, see below. Note that we estimate an average uncertainty of $5 \%$ in all theoretical calculations of the inclusive charged hadron cross sections stemming from the propagation of uncertainties of pion, kaon, and proton fragmentation functions. This additional uncertainty is included in the $\chi^{2}$ minimization procedure for determining $D_{i}^{r e s^{ \pm}}$.

As can be inferred from Tab. IV our global analysis contains also several other data sets which help to further constrain certain aspects of the residual charged hadron fragmentation functions and, more importantly, provide additional consistency checks. Longitudinal cross section measurements in $e^{+} e^{-}$annihilation are expected to help to constrain gluon fragmentation since, contrary to in-
TABLE III: Parameters describing the NLO fragmentation functions for positively charged residual hadrons, $D_{i}^{r e s+}\left(z, \mu_{0}\right)$, at the input scale $\mu_{0}=1 \mathrm{GeV}$. Inputs for the charm and bottom fragmentation functions refer to $\mu_{0}=$ $m_{c}=1.43 \mathrm{GeV}$ and $\mu_{0}=m_{b}=4.3 \mathrm{GeV}$, respectively.

\begin{tabular}{cccccc}
\hline \hline flavor $i$ & $N_{i}$ & $\alpha_{i}$ & $\beta_{i}$ & $\gamma_{i}$ & $\delta_{i}$ \\
\hline$u+\bar{u}$ & 0.0038 & 10.000 & 1.20 & 0.0003 & 18.51 \\
$\bar{u}$ & 0.0001 & 10.000 & 21.20 & 0.0003 & 18.51 \\
$c+\bar{c}$ & 0.0752 & 0.406 & 3.91 & 0.0000 & 0.00 \\
$b+\bar{b}$ & 0.0936 & -0.150 & 3.61 & 0.0000 & 0.00 \\
$g$ & 0.0001 & -0.497 & 9.99 & 20.000 & 14.75 \\
\hline \hline
\end{tabular}

TABLE IV: Data used in the NLO global analysis of residual charged hadron fragmentation functions, the individual $\chi^{2}$ values for each set, the fitted normalizations, and the total $\chi^{2}$ of the fit.

\begin{tabular}{|c|c|c|c|c|}
\hline experiment & $\begin{array}{l}\text { data } \\
\text { type }\end{array}$ & $\begin{array}{l}\text { rel. norm. } \\
\text { in fit }\end{array}$ & $\begin{array}{c}\text { data points } \\
\text { fitted }\end{array}$ & $\chi^{2}$ \\
\hline $\mathrm{TPC}[2]$ & incl. & 1.027 & 17 & 1.7 \\
\hline SLD [3] & incl. & 1.006 & 21 & 13.2 \\
\hline ALEPH [4] & incl. & 1.027 & 27 & 27.0 \\
\hline \multirow[t]{3}{*}{ DELPHI [5] } & incl. & 1.0 & 12 & 6.8 \\
\hline & "uds tag" & 1.0 & 12 & 7.6 \\
\hline & "b tag" & 1.0 & 12 & 4.9 \\
\hline \multirow[t]{2}{*}{ TASSO [11] } & incl. $(44 \mathrm{GeV})$ & 1.0 & 14 & 11.5 \\
\hline & incl. $(35 \mathrm{GeV})$ & 1.0 & 14 & 19.9 \\
\hline \multirow[t]{4}{*}{ OPAL [12] } & incl. & 1.0 & 12 & 5.7 \\
\hline & "uds tag" & 1.0 & 12 & 11.9 \\
\hline & "c tag" & 1.0 & 12 & 7.4 \\
\hline & "b tag" & 1.0 & 12 & 3.9 \\
\hline ALEPH [4] & long. incl. & 1.0 & 11 & 1.8 \\
\hline OPAL [13] & long. incl. & 1.0 & 12 & 3.3 \\
\hline \multirow[t]{3}{*}{ DELPHI [14] } & long. incl. & 1.0 & 12 & 11.6 \\
\hline & long. "uds tag" & 1.0 & 12 & 35.1 \\
\hline & long. "b tag" & 1.0 & 12 & 4.7 \\
\hline \multirow[t]{2}{*}{ EMC [18] } & $h^{+}$ & 0.987 & 98 & 99.1 \\
\hline & $h^{-}$ & 0.987 & 99 & 156.8 \\
\hline \multirow[t]{2}{*}{$\mathrm{CDF}$ [15] } & $630 \mathrm{GeV}$ & 1.1 & 16 & 103.4 \\
\hline & $1.8 \mathrm{TeV}$ & 1.1 & 37 & 112.7 \\
\hline \multirow[t]{4}{*}{ UA1 [16] } & $200 \mathrm{GeV}$ & 1.1 & 31 & 111.5 \\
\hline & $500 \mathrm{GeV}$ & 1.1 & 32 & 44.5 \\
\hline & $630 \mathrm{GeV}$ & 1.1 & 41 & 214.2 \\
\hline & $900 \mathrm{GeV}$ & 1.1 & 44 & 118.1 \\
\hline UA2 [17] & $540 \mathrm{GeV}$ & 1.1 & 27 & 89.3 \\
\hline TOTAL: & & & 661 & 1227.6 \\
\hline
\end{tabular}

clusive total cross sections, a non-vanishing $\mathcal{O}\left(\alpha_{s}\right)$ gluon coefficient function is already present at LO. For our NLO fits of the longitudinal cross section, we include the $\mathcal{O}\left(\alpha_{s}^{2}\right)$ coefficient functions [24]. In Fig. 8 we compare the result from the NLO global analysis for the longitudinal inclu- 


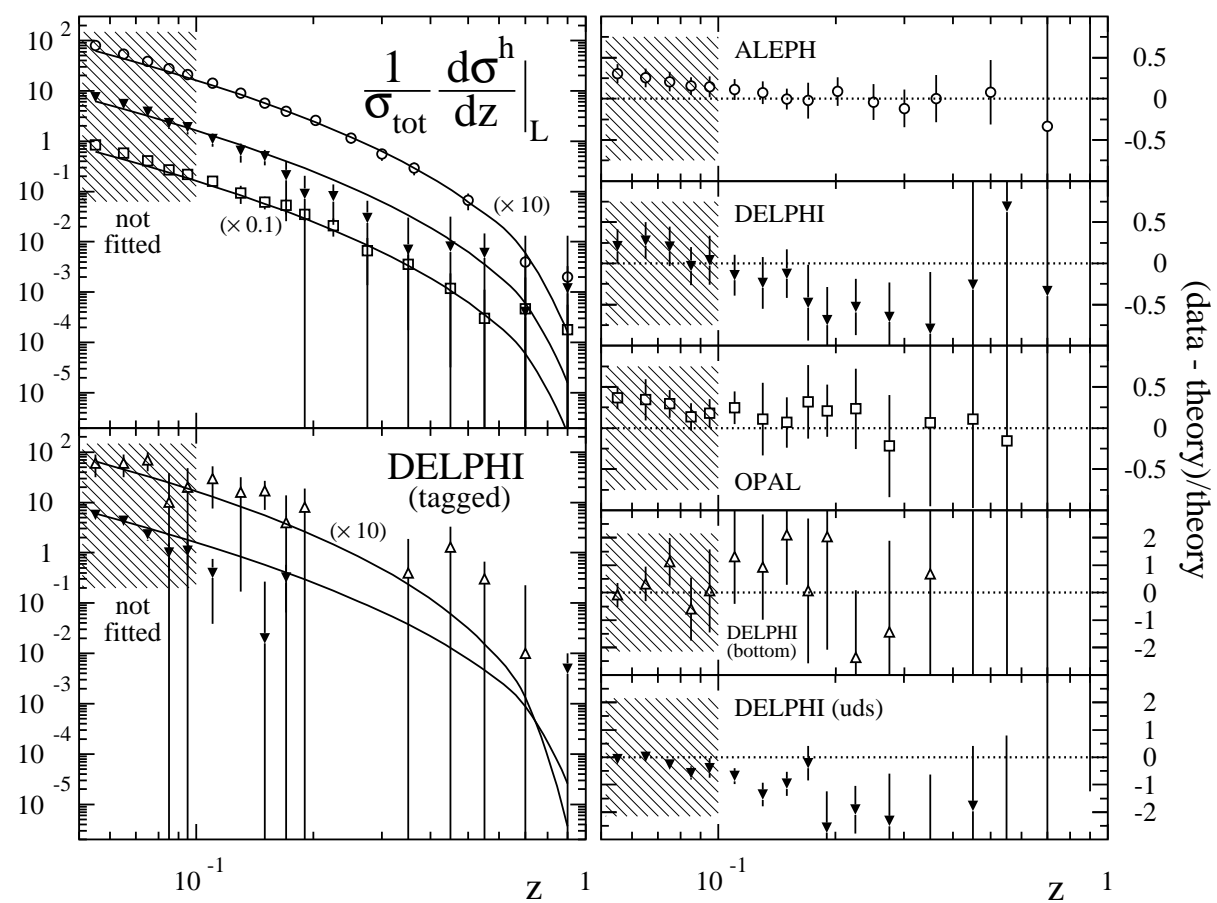

FIG. 8: Same as in Figs. 6 and 7 but now for the longitudinal cross sections.

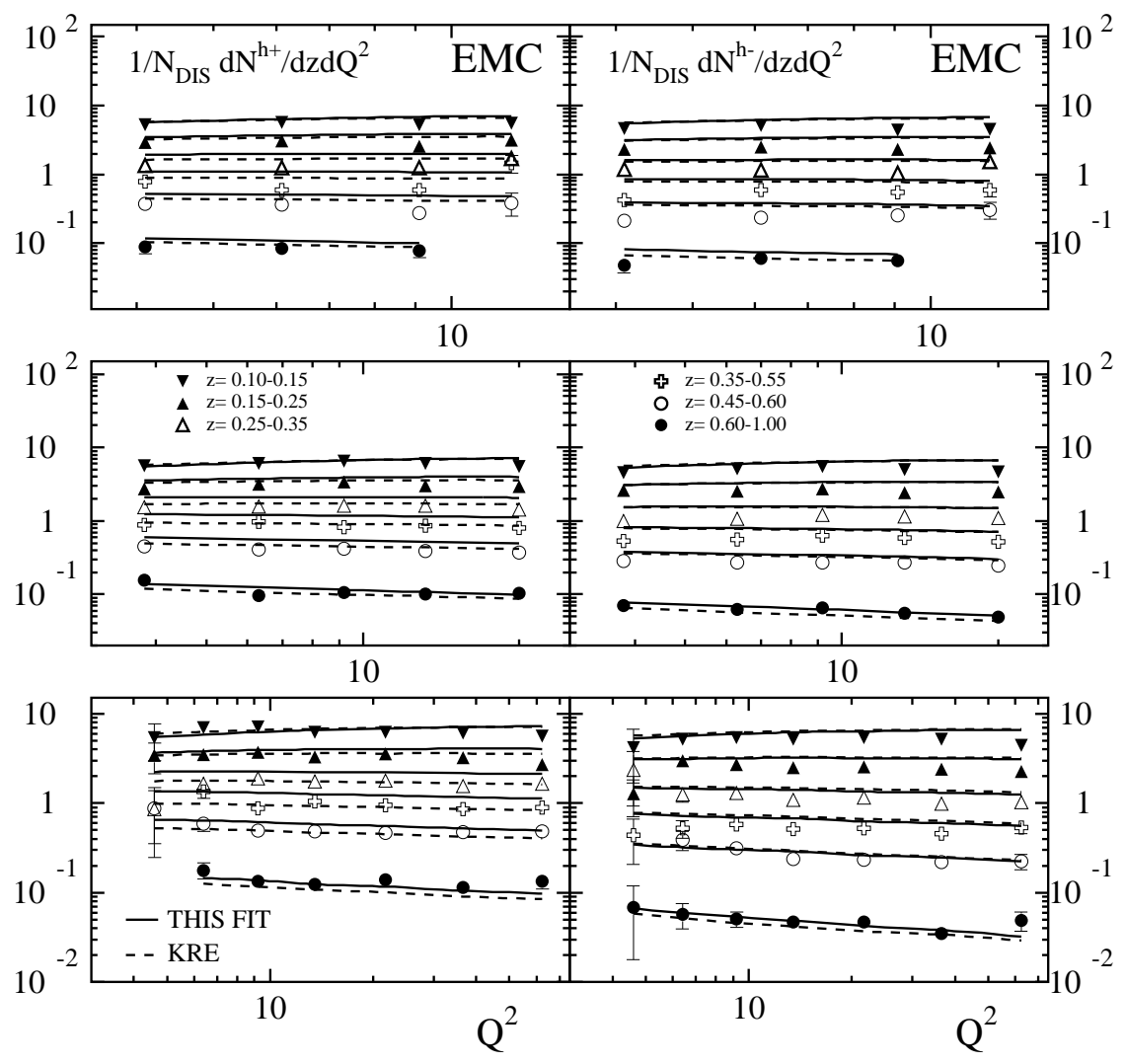

FIG. 9: Comparison of our NLO results (solid lines) for the positively and negatively charged hadron multiplicities in SIDIS, $\left(1 / N_{\text {DIS }}\right) d N^{h^{ \pm}} / d z d Q^{2}$, with data from EMC [18] for different bins in $z$. Upper, middle and lower panels correspond to beam energies of 120, 200, and $280 \mathrm{GeV}$, respectively. Also shown are the results obtained with the KRE [19] parameterization (dashed lines). 

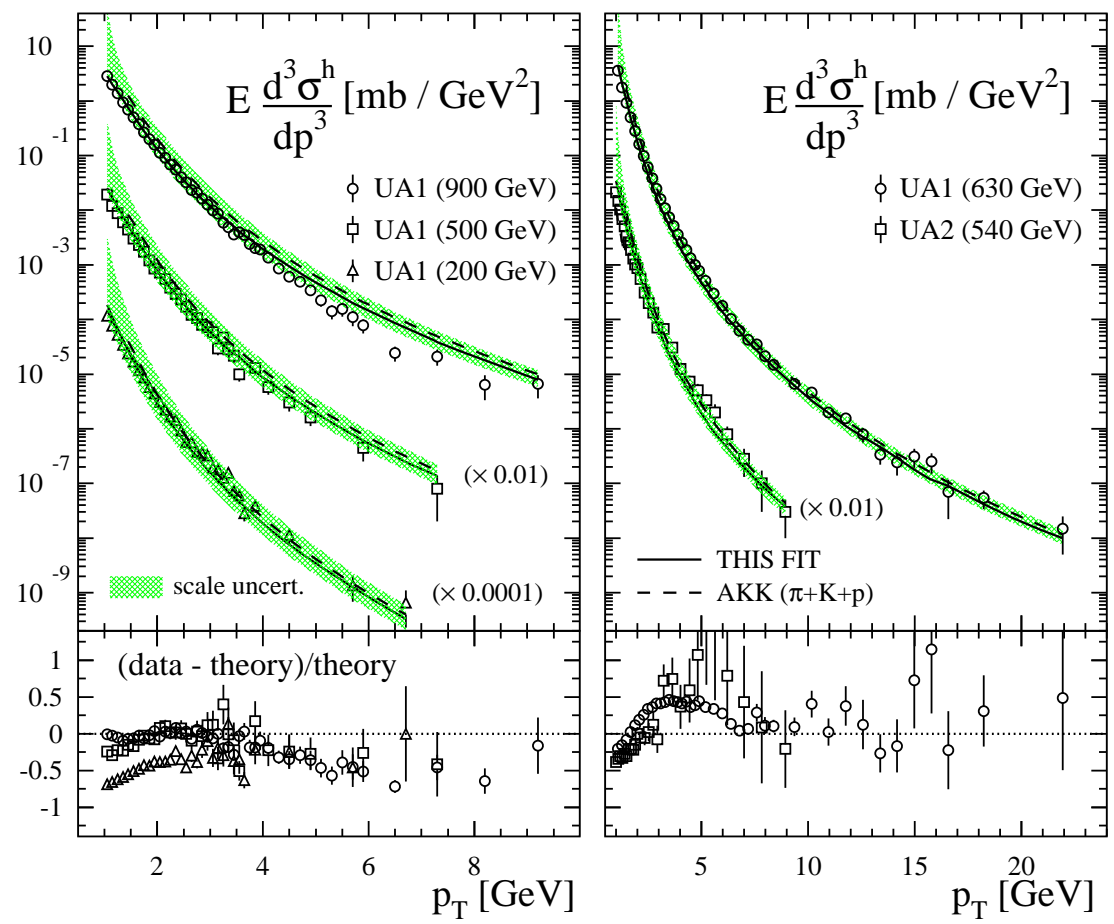

FIG. 10: Upper panels: comparison of our NLO results for single-inclusive charged hadron production $p \bar{p} \rightarrow h X$, where $h=\left(h^{+}+h^{-}\right) / 2$, with data from UA1 [16] and UA2 [17] for various c.m.s. energies $\sqrt{s}$ using $\mu_{f}=\mu_{r}=p_{T}$. The UA1 data cover $|y|<2.5$, except for $630 \mathrm{GeV}$, where $|y|<3.0$ and also $h=\left(h^{+}+h^{-}\right)$. For UA2 the rapidity range $1.0 \leq|y| \leq 1.8$ is covered. Also shown are the results obtained with the AKK [8] parametrization including only pions, kaons, and (anti-)protons. The shaded bands indicate theoretical uncertainties when all scales are varied in the range $p_{T} / 2 \leq \mu_{f}=\mu_{r} \leq 2 p_{T}$. Lower panels: "(data-theory)/theory" for our NLO results.

sive and flavor tagged cross section with the available LEP data [4, 13, 14]. With the exception of the ALEPH data, the statistical precision is not really impressive, in particular, in the fitted region $z>z_{\min }$, but all data are reasonably well described by the fit.

Data on charged pion and kaon multiplicities in SIDIS have provided valuable information on scale dependence and the charge and flavor separation of the corresponding fragmentation functions in our previous analysis [1]. For inclusive charged hadrons, the data collected by EMC [18] cover a rather large kinematic region with very narrow $Q^{2}$-bins, what makes it particularly suitable for a QCD fit. As expected, these data prove to be very valuable in our global analysis here. Figure 9 shows the outcome of the fits compared to the EMC multiplicities for positively and negatively charged hadrons covering all beam energies of 120,200 , and $280 \mathrm{GeV}$. The good precision of the data over the entire $z$ range helps to further constrain the high $z$ behavior of the quark and anti-quark fragmentation functions. An interesting thing to notice is that even though the KRE sets for pions and kaons overestimate the corresponding SIDIS multiplicities as demonstrated in [1], this effect is somehow compensated for in the charged hadron multiplicities shown in Fig. 9 at the expense of comparatively small residual fragmentation functions. In order to account for the error introduced by the finite size of the bins in $z$ and $Q^{2}$, as well as the uncertainties introduced by the use of the pion, kaon, and proton fragmentation functions, we include an additional $10 \%$ theoretical uncertainty in the $\chi^{2}$ minimization. There is also an additional $11 \%$ experimental normalization uncertainty [18] not included in the errors bars shown in Fig. 9.

The final ingredient to our global analysis of fragmentation functions $D_{i}^{h^{ \pm}}$for unidentified charged hadrons are single-inclusive hadroproduction data from $p \bar{p}$ collisions measured by the UA1 [16] and UA2 [17] collaborations at CERN, and by the CDF collaboration [15] at Fermilab's TeVatron. We have not included proton-proton collision data from fixed target experiments, since the validity of fixed order calculations in $\mathrm{PQCD}$ at lower energies is seriously in doubt 25]. The data span a range of c.m.s. energies $\sqrt{s}$ from $200 \mathrm{GeV}$ to $1.8 \mathrm{TeV}$ but do not discriminate different hadron charges. Due to the dominance of gluoninduced processes at the available small-to-medium values of the hadron's $p_{T}$, they mainly probe the gluon fragmentation function $D_{g}^{h^{ \pm}}$. To give an example, in the case of UA1 at $\sqrt{s}=630 \mathrm{GeV}$, where the available data cover the largest range of transverse momenta $p_{T}$ from $1 \mathrm{GeV}$ to $22 \mathrm{GeV}$, in which the gluonic contribution decreases from around $90 \%$ in the lowest $p_{T}$ bin to a sizable $40 \%$ at the highest transverse momentum. Figures 10 and 11 show the comparisons between the results of our NLO fits to the single-inclusive cross sections and the experimen- 


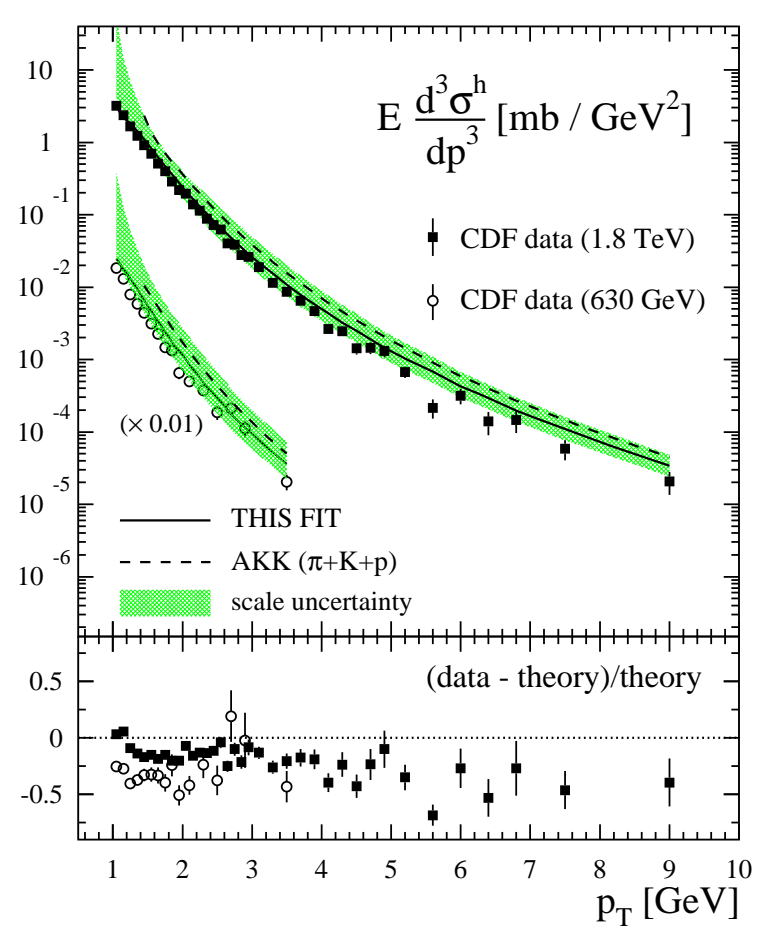

FIG. 11: Similar to Fig. 10 but now comparing our NLO results to the CDF data at two different c.m.s. energies $\sqrt{s}$. The data are for $h=\left(h^{+}+h^{-}\right) / 2$ and cover $|y| \leq 1$ in rapidity.

tal data from CERN and Fermilab, respectively. Within the fairly large theoretical scale ambiguities, indicated by the shaded bands, which refer to varying the factorization and renormalization scales simultaneously in the range $p_{T} / 2 \lesssim \mu_{f}=\mu_{r} \lesssim 2 p_{T}$, the overall agreement between theory and data is reassuringly good. The obtained gluon fragmentation functions into charged pions, kaons, and (anti-)protons, which dominate the single-inclusive cross sections shown in Figs. 10 and 11, are consistent with data, and the residual contribution $D_{g}^{\text {res }}{ }^{ \pm}$is very small. Again, we include a 5\% theoretical error due to the choice of the scale in the $\chi^{2}$ minimization and another $5 \%$ associated with the propagation of the uncertainties of pion, kaon, and proton fragmentation functions.

The resulting fragmentation functions for positively charged hadrons $D_{i}^{h^{+}}$, evolved to the scale $M_{Z}$, are shown in Fig. 12 and compared to the distributions of KRE [19] and AKK [8]. Recall that in the AKK analysis $D_{i}^{h^{+}}$ is approximated by the sum of pion, kaon, and proton fragmentation functions. Compared to sets of KRE and AKK, we find again good agreement in the total singlet contribution $D_{\Sigma}^{h^{+}}$, but some noticeable differences in the charge and flavor separation, for instance, in $D_{\bar{u}}^{h^{+}}$, and in the gluon fragmentation $D_{g}^{h^{+}}$. In general, the differences become larger towards $z \rightarrow 1$, which has been already observed for the pion, kaon [1], and proton fragmentation functions (Fig. 44).

One striking feature of the distributions shown in Fig. 12 is the only slightly broken flavor democracy for

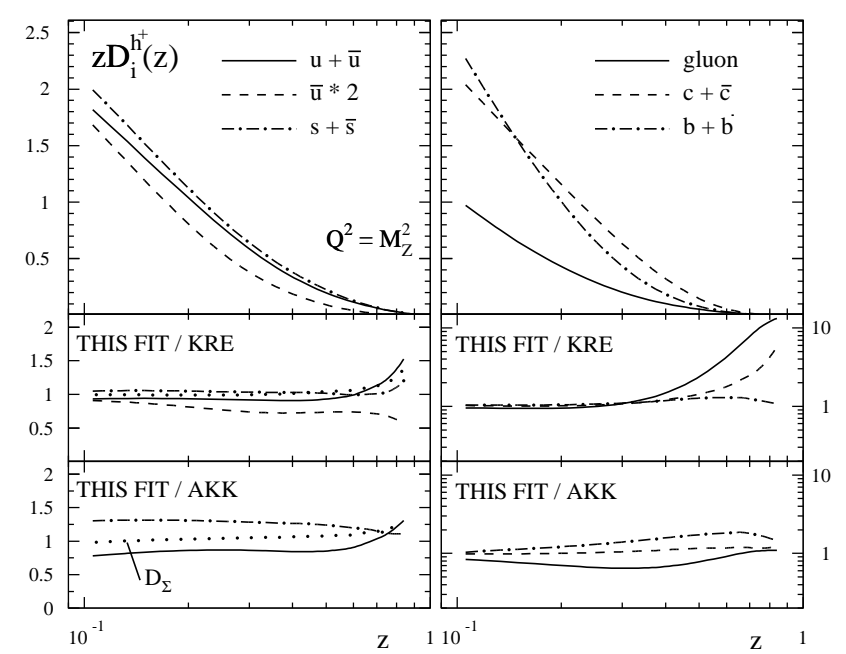

FIG. 12: Upper panels: individual fragmentation functions for positively charged hadrons $z D_{i}^{h^{+}}\left(z, Q^{2}\right)$ at $Q^{2}=M_{Z}^{2}$ for $i=u+\bar{u}, 2 \bar{u}, s+\bar{s}, g, c+\bar{c}$, and $b+\bar{b}$. Middle panels: ratios of our fragmentation functions to the ones of KRE [19]. The dotted line indicates the ratio for singlet combination of fragmentation functions $z D_{\Sigma}^{h^{+}}$. Lower panels: ratios of our fragmentation functions to the ones of AKK [8]; note that $D_{\bar{u}}^{h^{+}}$is not available in the AKK analysis.

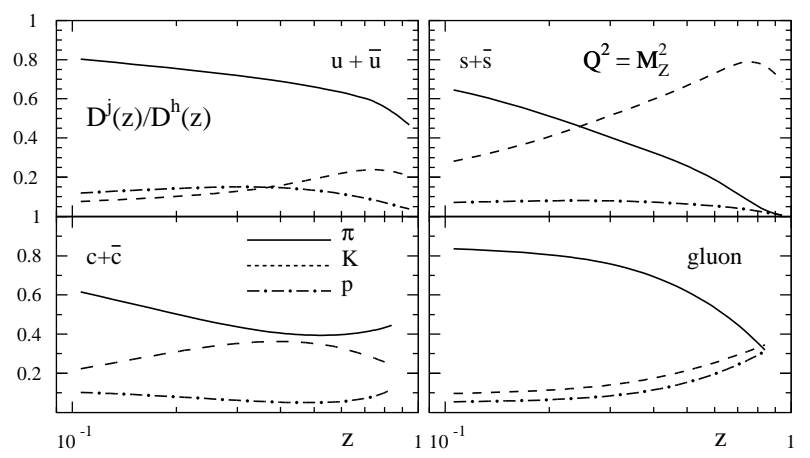

FIG. 13: Partial contributions of pions, kaons, and protons to the charged hadron fragmentation functions as a function of $z$ at $Q^{2}=M_{Z}^{2}$.

$q+\bar{q}$ fragmentation functions into charged hadrons. To understand this better, the partial contributions of pions, kaons, and protons to the charged hadron fragmentation functions are shown in Fig. 13. As can be noticed, the $u$ quark and gluon fragmentation functions are completely dominated by the pion contribution, except for very large momentum fractions $z$, where proton-proton scattering data require some increase in the kaon and proton part. The strange quark fragmentation function for charged hadrons in the valence region is, as expected, dominated by kaons, while both kaons and pions contribute with a similar amount to the charm (and bottom) distributions. The residual contribution becomes sizable only for the heavy quark fragmentation functions.

Even though the relative uncertainties of the residual 

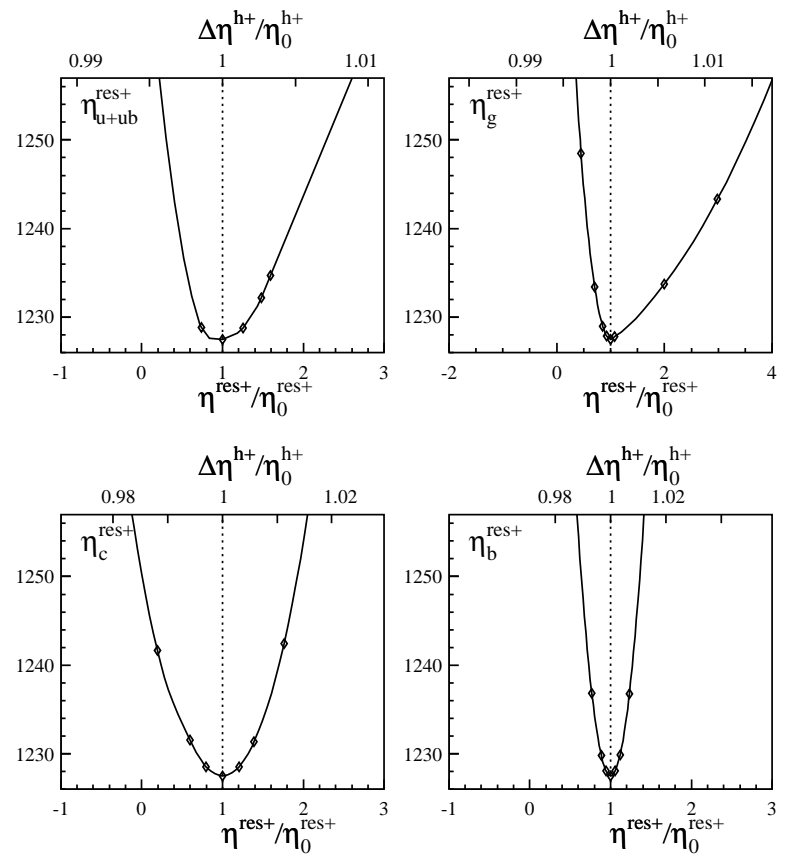

FIG. 14: Profiles of $\chi^{2}$ for the NLO residual charged hadron fragmentation fit as a function of the truncated second moments $\eta_{i}^{\text {res }}{ }^{+}\left(x_{p}=0.2, Q^{2}=50 \mathrm{GeV}^{2}\right)$ for different flavors. The moments are normalized to the value $\eta_{i 0}^{\text {res }^{+}}$they take in the best fit to data. The upper horizontal scales show variations relative to the change in the moments of the total charged hadron fragmentation functions $\eta^{h^{+}}$.

charged hadron fragmentation functions $D_{i}^{r e s^{ \pm}}$are rather large, exceeding even $200 \%$ within a conservative $2 \%$ increase $\Delta \chi^{2}$ in the total $\chi^{2}$ of the fit, the effect on $D_{i}^{h+}$ is fairly small and amounts to less than about $2 \%$. This is readily explained by the relative smallness of the residual charged hadron contribution to the sum in Eq. (17). The best constrained residual densities are those for charm and bottom, which are also the most significant ones in absolute size, although almost innocuous for SIDIS and $p \bar{p}$ data. In Fig. 14 we show the $\chi^{2}$-profiles of the global fit as a function of the range of variation of the truncated second moments of the residual charged hadron fragmentation functions $D_{i}^{r e s^{+}}$, see Eq. (12). In the upper horizontal scales we also show the impact of those variations relative to the change in the moments of the total charged hadron fragmentation functions $D_{i}^{h^{+}}$.

\section{CONCLUSIONS}

By extending our previous global analyses for pions and kaons to the case of (anti-)protons and unidentified charged hadrons, we have completed a comprehensive study of single-inclusive hadron production within pQCD at NLO accuracy. Specifically, we have demonstrated that the pQCD framework based on factorized cross sections can consistently account for a large variety
TABLE V: As in Tab. \but now describing the LO fragmentation functions into protons, $D_{i}^{p}\left(z, \mu_{0}\right)$, at the input scale.

\begin{tabular}{cccccc}
\hline \hline flavor $i$ & $N_{i}$ & $\alpha_{i}$ & $\beta_{i}$ & $\gamma_{i}$ & $\delta_{i}$ \\
\hline$u+\bar{u}$ & 0.094 & 0.041 & 1.485 & 15.0 & 3.44 \\
$d+\bar{d}$ & 0.059 & 0.041 & 1.485 & 15.0 & 3.44 \\
$\bar{u}$ & 0.036 & 0.041 & 1.998 & 15.0 & 3.44 \\
$\bar{d}$ & 0.022 & 0.041 & 1.998 & 15.0 & 3.44 \\
$s+\bar{s}$ & 0.045 & 0.041 & 1.998 & 15.0 & 3.44 \\
$c+\bar{c}$ & 0.079 & -0.887 & 5.436 & 0.0 & 0.00 \\
$b+\bar{b}$ & 0.047 & 0.103 & 10.00 & 0.0 & 0.00 \\
$g$ & 0.029 & 6.000 & 1.200 & 0.0 & 0.000 \\
\hline \hline
\end{tabular}

TABLE VI: As in Tab. III but now for the LO fragmentation functions into positively charged residual hadrons $D_{i}^{\text {res }}{ }^{+}$.

\begin{tabular}{cccccc}
\hline \hline flavor $i$ & $N_{i}$ & $\alpha_{i}$ & $\beta_{i}$ & $\gamma_{i}$ & $\delta_{i}$ \\
\hline$u+\bar{u}$ & 0.0002 & 9.986 & 1.543 & 15.0 & 19.90 \\
$\bar{u}$ & 0.0001 & 9.986 & 21.543 & 15.0 & 19.90 \\
$c+\bar{c}$ & 0.147 & -0.051 & 2.792 & 0.0 & 0.00 \\
$b+\bar{b}$ & 0.113 & -0.574 & 2.949 & 0.0 & 0.00 \\
$g$ & 0.0001 & -0.499 & 10.000 & 20.0 & 19.64 \\
\hline \hline
\end{tabular}

TABLE VII: Same as in Tab. [I] but now at LO accuracy.

\begin{tabular}{lcccc}
\hline \hline experiment & data & rel. norm. & data points & $\chi^{2}$ \\
& type & in fit & fitted & \\
\hline TPC [2] & incl. & 1.043 & 12 & 7.5 \\
SLD [3] & incl. & 0.983 & 18 & 11.8 \\
& "uds tag" & 0.983 & 9 & 10.7 \\
& "c tag" & 0.983 & 9 & 9.6 \\
& "b tag" & 0.983 & 9 & 9.3 \\
ALEPH [4] & incl. & 0.97 & 13 & 11.6 \\
DELPHI [5] & incl. & 1.0 & 12 & 3.9 \\
& "uds tag" & 1.0 & 12 & 0.7 \\
& "b tag" & 1.0 & 12 & 9.0 \\
OPAL [6] & " $u$ tag" & 1.10 & 5 & 7.8 \\
& " $d$ tag" & 1.10 & 5 & 12.8 \\
& "s tag" & 1.10 & 5 & 5.4 \\
& "c tag" & 1.10 & 5 & 5.0 \\
& "b tag" & 1.10 & 5 & 5.7 \\
\hline STAR [10] & $p$ & 0.95 & 14 & 42.9 \\
& $\bar{p}$ & 0.95 & 14 & 32.4 \\
\hline \hline TOTAL: & & & 159 & 186.1 \\
\hline \hline
\end{tabular}

of processes with hadrons in the final-state with remarkable precision, producing at the same time accurate and universal sets of fragmentation functions. The availability of these crucial non-perturbative inputs now opens a wide range of possibilities for detailed studies of the nu- 
TABLE VIII: Same as in Tab. IV but now at LO accuracy.

\begin{tabular}{|c|c|c|c|c|}
\hline experiment & $\begin{array}{l}\text { data } \\
\text { type }\end{array}$ & $\begin{array}{l}\text { rel. norm. } \\
\text { in fit }\end{array}$ & $\begin{array}{c}\text { data points } \\
\text { fitted }\end{array}$ & $\chi^{2}$ \\
\hline $\mathrm{TPC}[2]$ & incl. & 1.041 & 17 & 7.7 \\
\hline SLD [3] & incl. & 1.014 & 21 & 63.0 \\
\hline ALEPH [4] & incl. & 1.013 & 27 & 46.4 \\
\hline \multirow[t]{3}{*}{ DELPHI [5] } & incl. & 1.0 & 12 & 12.2 \\
\hline & "uds tag" & 1.0 & 12 & 22.3 \\
\hline & "b tag" & 1.0 & 12 & 16.6 \\
\hline \multirow[t]{2}{*}{ TASSO [11] } & incl. $(44 \mathrm{GeV})$ & 1.0 & 14 & 22.3 \\
\hline & incl. $(35 \mathrm{GeV})$ & 1.0 & 14 & 56.3 \\
\hline \multirow[t]{4}{*}{ OPAL [12] } & incl. & 1.0 & 12 & 28.5 \\
\hline & "uds tag" & 1.0 & 12 & 32.9 \\
\hline & "c tag" & 1.0 & 12 & 18.1 \\
\hline & "b tag" & 1.0 & 12 & 8.6 \\
\hline ALEPH [4] & long. incl. & 1.0 & 11 & 11.5 \\
\hline OPAL [13] & long. incl. & 1.0 & 12 & 8.1 \\
\hline \multirow[t]{3}{*}{ DELPHI [14] } & long. incl. & 1.0 & 12 & 7.2 \\
\hline & long. " $u d s$ tag" & 1.0 & 12 & 28.0 \\
\hline & long. "b tag" & 1.0 & 12 & 5.0 \\
\hline \multirow[t]{2}{*}{ EMC [18] } & $h^{+}$ & 1.0 & 98 & 183.0 \\
\hline & $h^{-}$ & 1.0 & 99 & 289.1 \\
\hline \multirow[t]{2}{*}{ CDF [15] } & $630 \mathrm{GeV}$ & 1.1 & 16 & 222.4 \\
\hline & $1.8 \mathrm{TeV}$ & 1.1 & 37 & 515.5 \\
\hline \multirow[t]{4}{*}{ UA1 [16] } & $200 \mathrm{GeV}$ & 0.988 & 31 & 84.5 \\
\hline & $500 \mathrm{GeV}$ & 0.988 & 32 & 129.4 \\
\hline & $630 \mathrm{GeV}$ & 0.988 & 41 & 48.3 \\
\hline & $900 \mathrm{GeV}$ & 0.988 & 44 & 391.7 \\
\hline UA2 [17] & $540 \mathrm{GeV}$ & 0.988 & 27 & 135.3 \\
\hline TOTAL: & & & 661 & 2393.9 \\
\hline
\end{tabular}

cleon structure at NLO accuracy through single-inclusive hadroproduction processes, encompassing the study of the spin and flavor structure with polarized SIDIS measurements [26], in proton-proton collisions [27], and in photoproduction [28], the modification of parton densities by nuclear effects 29], or studies of high $p_{T}$ electroproduction processes [30].

\section{Acknowledgments}

We warmly acknowledge Werner Vogelsang for helpful discussions, and Carlos García Canal for comments and suggestions. This work was partially supported by CONICET, ANPCyT and UBACyT.

\section{APPENDIX: LO RESULTS}

For completeness, we have also performed global analyses of the same sets of data given in Tables II and IV. where now all observables, $\alpha_{s}$, and the scale evolution of the fragmentation functions are computed at LO accuracy. We use the same parametrization (11) and fitting procedure as in the NLO case, outlined in Sec. II. The parameters of the optimum LO fits for $D_{i}^{p}$ and $D_{i}^{r e s^{+}}$are given in Tables $\mathrm{V}$ and $\mathrm{VI}$ while the $\chi^{2}$ values obtained for the individual sets of data are compiled in Tables VII and VIII, respectively.

It is important to notice that the total $\chi^{2}$ of the LO fits is significantly worse than at NLO accuracy, in particular, in case of the inclusive charged hadron data. Our LO sets should be used only for rough estimates of observables where NLO corrections are not yet available, or in event generators based on matrix elements at LO accuracy. Because of the limited usefulness of the LO sets, we refrain from going into any further details here.
[1] D. de Florian, R. Sassot and M. Stratmann, Phys. Rev. D75, 114010 (2007).

[2] H. Aihara et al. (TPC Collaboration), Phys. Rev. Lett. 61, 1263 (1998); Phys. Lett. B184, 299 (1987); X.-Q. Lu, Ph.D. thesis, Johns Hopkins University [Report No. UMI-87-07273, 1986].

[3] K. Abe et al. (SLD Collaboration), Phys. Rev. D59, 052001 (1999).

[4] D. Buskulic et al. (ALEPH Collaboration), Z. Phys. C66, 355 (1995); Phys. Lett. B357, 487 (1995); R. Barate et al. (ALEPH Collaboration), Phys. Rept. 294, 1 (1998).

[5] P. Abreu et al. (DELPHI Collaboration), Eur. Phys. J. C5, 585 (1998).

[6] G. Abbiendi et al. (OPAL Collaboration), Eur. Phys. J. C16, 407 (2000).

[7] B.A. Kniehl, G. Kramer, and B. Pötter, Nucl. Phys. B582, 514 (2000).

[8] S. Albino, B.A. Kniehl, and G. Kramer, Nucl. Phys.
B725, 181 (2005); B734, 50 (2006).

[9] M. Hirai, S. Kumano, T.-H. Nagai, and K. Sudoh, Phys. Rev. D75, 094009 (2007)

[10] J. Adams et al. (STAR Collaboration), Phys. Lett. B637, 161 (2006).

[11] W. Braunschweig et al. (TASSO Collaboration), Z. Phys. C42, 189 (1989).

[12] K. Ackerstaff et al. (OPAL Collaboration), Eur. Phys. J. C7, 369 (1999).

[13] R. Akers et al. (OPAL Collaboration), Z. Phys. C68, 203 (1995).

[14] P. Abreu et al. (DELPHI Collaboration), Eur. Phys. J. C6, 19 (1999).

[15] F. Abe et al. (CDF Collaboration), Phys. Rev. Lett. 61, 1819 (1988).

[16] C. Albajar et al. (UA1 Collaboration), Nucl. Phys. B335, 261 (1990); G. Bocquet et al. (UA1 Collaboration), Phys. Lett. B366, 434 (1996). 
[17] M. Banner et al. (UA2 Collaboration), Z. Phys. C27, 329 (1985).

[18] J. Ashman et al. (EMC Collaboration), Z. Phys. C52, 361 (1991).

[19] S. Kretzer, Phys. Rev. D62, 054001 (2000).

[20] L. Bourhis, M. Fontannaz, J. Ph. Guillet, and M. Werlen, Eur. Phys. J. C19, 89 (2001).

[21] M. Stratmann and W. Vogelsang, Phys. Rev. D64, 114007 (2001).

[22] I. Arsene et al. (BRAHMS Collaboration), arXiv:hep-ex/0701041.

[23] D. Stump et al., Phys. Rev. D65, 014012 (2002).

[24] P.J. Rijken and W.L. van Neerven, Nucl. Phys. B487, 233 (1997); Phys. Lett. B386, 422 (1996); A. Mitov and S.O. Moch, Nucl. Phys. B751, 18 (2006).

[25] D. de Florian and W. Vogelsang, Phys. Rev. D71, 114004
(2005).

[26] D. de Florian, G.A. Navarro, and R. Sassot, Phys. Rev. D71, 094018 (2005).

[27] D. de Florian, Phys. Rev. D67, 054004 (2003); B. Jäger, A. Schäfer, M. Stratmann, and W. Vogelsang, Phys. Rev. D67, 054005 (2003).

[28] B. Jäger, M. Stratmann, and W. Vogelsang, Phys. Rev. D68, 114018 (2003); Eur. Phys. J. C44, 533 (2005).

[29] D. de Florian and R. Sassot, Phys. Rev. D69, 074028 (2004).

[30] A. Daleo, D. de Florian, and R. Sassot, Phys. Rev. D71, 034013 (2005).

[31] A Fortran package containing our LO and NLO sets of fragmentation functions can be obtained upon request from the authors. 